ORIGINAL ARTICLE

\title{
Readiness of Health Centers and Primary Hospitals for the Implementation of Proposed Health Insurance Schemes in Southwest Ethiopia
}

\author{
Sabit Abazinab ${ }^{1}$, Mirkuzie Woldie ${ }^{2}$, Tesfamichael Alaro ${ }^{2}$
}

ABSTRACT

BACKGROUND: In response to the 2005 World Health Assembly, many low income countries developed different healthcare financing mechanisms with risk pooling stategy to ensure universal coverage of health services. Accordingly, service availability and readiness of the health system to bear the responsibility of providing service have critical importance. The objective of this study was to assess service availability and readiness of health centers and primary hospitals to bear the responsibility of providing service for the members of health insurance schemes.

METHODS AND MATERIALS: A facility based cross sectional study design with quantitative data collection methods was employed. Of the total 18 districts in Jimma Zone, 6(33.3\%) districts were selected randomly. In the selected districts, there were 21 functional public health facilities (health centers and primary hospitals) which were included in the study. Data were collected by interviewer administered questionnaire. Descriptive statistics were calculated by using SPSS version 20.0. Prior to data collection, ethical clearance was obtained.

RESULTS: Among the total 21 public health facilities surveyed, only $38.1 \%$ had all the categories of health professionals as compared to the national standards. The majority, 85.2\%, of the facilities fulfilled the criteria for basic equipment, but $47.7 \%$ of the facilities did not fulfill the criteria for infection prevention supplies. Moreover, only two facilities fulfilled the criteria for laboratory services, and $95.2 \%$ of the facilities had no units/departmenst to coordinate the health insurance schemes.

CONCLUSIONS: More than nine out of ten facilities did not fulfill the criteria for providing healthcare services for insurance beneficiaries and are not ready to provide general services according to the standard. Hence, policy makers and implementers should devise strategies to fill the identified gaps for successful and sustainable implementation of the proposed insurance scheme.

KEYWORDS: - Health Insurance,Public Health Facilities, Readiness

DOI: http://dx.doi.org/10.4314/ejhs.v26i5.6

\section{INTRODUCTION}

In response to the 2005 World Health Assembly, many developing countries of the world developed different risk pooling/prepayment mechanisms to ensure universal coverage of health services (1).
This will definitely reduce financial barriers, improve access to health services and increase health services utilizations at every level among the population, especially the poor $(2,3)$.

\footnotetext{
${ }^{1}$ Jimma Town Health office, Jimma, Ethiopia

${ }^{2}$ Department of Health Economics, Management and Policy, College of Health Sciences, Jimma University Corresponding Author: Tesfamichael Alaro, Email: tesfamichaelalaro@yahoo.com
} 
On the other hand, to handle the increased service utilization of the population, readiness of health facilities has a pivotal role. Readiness is defined as the cumulative availability of components required to provide services: infrastructure/amenities, basic supplies/equipment including small surgery, standard precautions, laboratory tests, medicines and commodities and health professionals (4).

The work force is central to advancing health, and there should be an optimum number and mix of professional (5). However, many countries across the globe are facing a challenge in meeting their required number of health care workers (6). For example, in the Philippines, healthcare providers were heavily concentrated in urban and wealthy communities $(7,8)$. The same trend was seen in Sub-Saharan Africa (SSA) countriesMozambique and Uganda $(9,10)$.

There is a wide gap in availability of basic amenities in healthcare facilities globally. About $38 \%$ of healthcare facilities do not have an improved water source, $19 \%$ do not provide improved sanitation, and $35 \%$ do not have soap for hand washing and $42 \%$ of all health care facilities lacking an improved water source in the African region (11).

Another study done in SSA on assessment of health facility readiness to provide family planning services showed that $90 \%$ the facilities met the criteria for infrastructure (12). Moreover, systematic review of available national data conducted on electricity access in health care facilities showed that, on average, $26 \%$ reported no access to electricity, only $28 \%$ of healthcare facilities had reliable electricity and an average of $7 \%$ of facilities relied solely on a generator (13).

In Uganda, less than $25 \%$ of the facilities had all essential equipment and supplies for basic antenatal care, while basic equipment and supplies for conducting normal deliveries were available in only $33 \%$ of the facilities (14). Another report also showed that over $74 \%$ of government health units reported monthly stock outs of tracer medicines (15). A study done in Sudan showed that, on average, availability of selected essential medicines at the public pharmacy was $80.6 \%$ (16).

In Ethiopia, a study done to estimate waste generation rate and evaluate its management system showed that only $40 \%$ used local type of incinerators, while others used open burning of healthcare wastes. Operational guidelines were not found in all assessed health centers (17).

Substantial investments have been and continue to be made to improve health services in Ethiopia. Sound decisions about where to invest more resources to improve health services require knowledge of the existing health system. In addition, improved health services will be delivered for insured population by health facilities with the required quality standards (18). However, information on the status of services and the overall health systems within which they operate is rare. Therefore, this study assessed the degree of readiness of primary hospitals and health centers in Jimma zone to implement the newly proposed health insurance schemes.

\section{METHODS}

A facility based cross sectional study was conducted using Service Availability and Readiness Assessment (SARA) questionnaire from March 01 to 30, 2015 in Jimma Zone, Southwest Ethiopia. The study included twenty one public health facilities from six randomly selected districts of Jimma Zone.The intervieweradministered questionnaire was developed and adopted with modification from related studies, national health facility standard manuals and WHO reference manuals (18-20). It has seven sections. The first section contains question to assess availability of health professionals. The second section contains questions to assess the availability of basic amenities, i.e. information communication technology (ICT) infrastructures, water supply and electricity and ambulance services. The third section contains questions to assess availability of basic equipment; the fourth section contains questions to assess the presence of standard precautions and supplies for infection prevention; the fifth section assesses laboratory capacity; the sixth section assesses the availability of essential medicines and the last section assess facility governing system. Furthermore. all sections have observation checklists.

Twelve diploma holding nurses participated in data collection, and six BSc holder health professionals supervised the process. Two interviewers were involved in data collection for each health institution to facilitate note-taking during observation. After the completion of the interview, the responses were cross checked 
through observation. The collected data were entered to Epi Data version 3.1, cleaned and cheeked for missing values; then exported to SPSS version 20.0 for analysis. Readiness of health facilities were evaluated in line with the Ethiopian Food, Medicine and Healthcare Administration and Control Authority (FMHACA) standard (18). Accordingly, overall facility readiness was assessed as good if the health facility fulfilled standards for staffing, and met $>=75 \%$ of the standards of infrastructures, basic equipment, infection prevention supplies, laboratory services and essential medicines, and had administrative arrangements to implement the health insurance scheme. Prior to data collection, ethical clearance was obtained from institutional review board of the College of Health
Sciences, Jimma University. Moreover, informed verbal consent was taken from the respondents.

\section{RESULTS}

In this study, a total of 21 sampled public health facilities were included. Among these, 18(85.7\%) were health centers and 3(4.3\%) were primary hospitals.

Staffing of health facilities: Among 3 primary hospitals surveyed, 1 hospital had only 2 general practitioners. However, all hospitals had more than 8 and 4 clinical nurses and laboratory professionals, respectively. Among 18 health centers surveyed, 4 facilities had no health officer/BSc nurse (Figure 1).

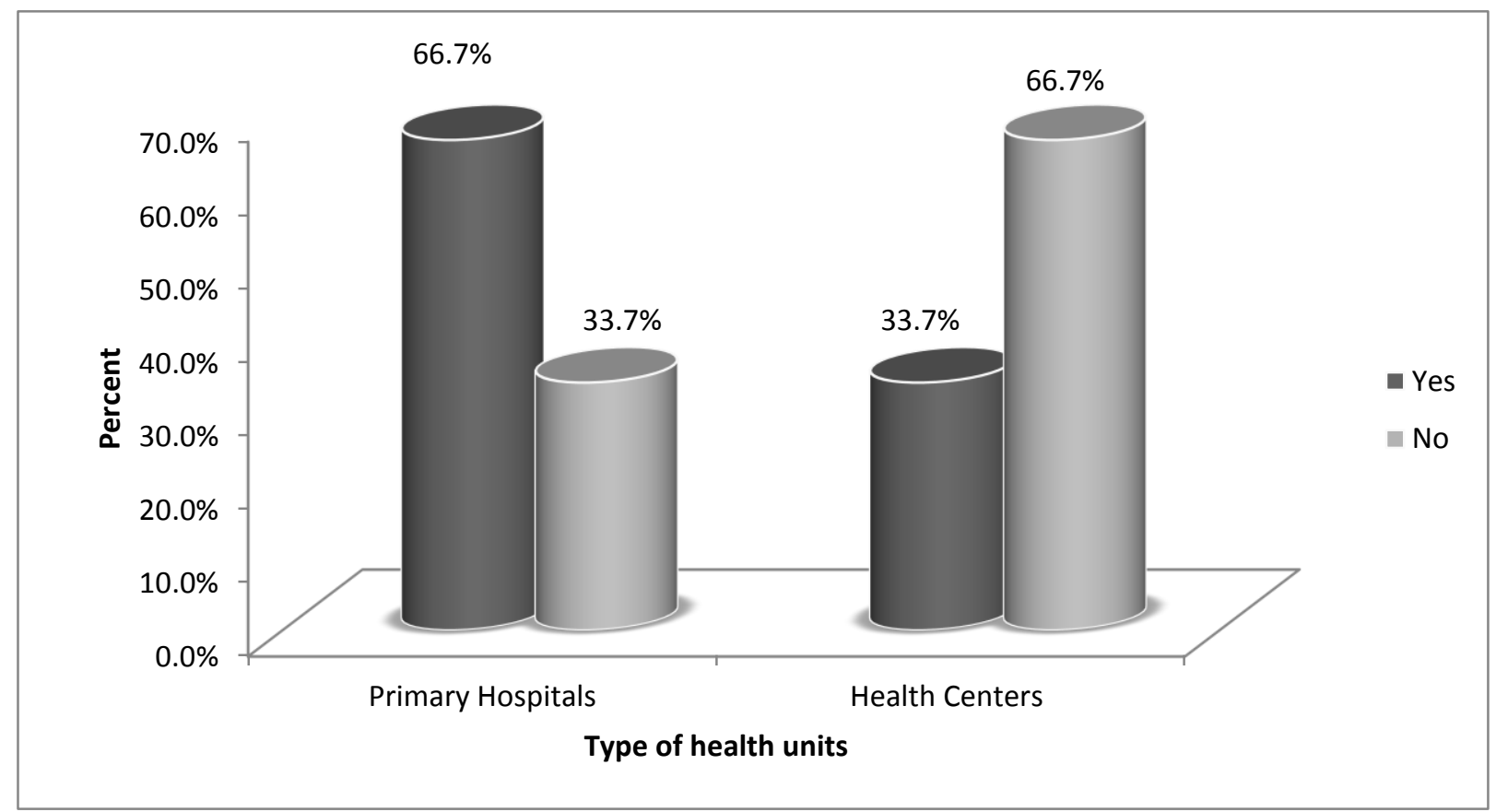

Figure 1: Proportion of health facilities that reported having health professionals according to the standards

Infrastructural amenities: Among 21 surveyed facilities, $8(38.1 \%)$ had no fixed line telephone or mobile service supported by the facility. All 21 facilities had desktop computers among which of only 2 had internet services. All of the facilities had electricity which was mainly from main electric lines. However, $14(66.7 \%)$ of the facilities had no generator. Among those 7 facilities which had generators as secondary source, only three of them had fuel on the date of the survey.

All of health facilities had water sources at less than 500 meters distance. More specifically, 5 facilities got water from protected well, and 16 from pipe. Ten $(47.6 \%)$ of the facilities had ambulances stationed at the facility, 5 had no ambulance services and 6 had ambulances services 
452 Ethiop J Health Sci.

from other areas/facilities. Sixteen (76.2\%) of the facilities had OPD which has auditory and visual privacy. Among all facilities $10(47.6 \%)$ had outpatient latrines; of which 4 were flush latrine, 6 were Ventilated Improved Pit (VIP) latrines with hand washing facilities.

Basic equipment: All health facilities surveyed had functional adult, child and infant weighing scale, measuring tape, and intravenous infusion kits. Despite that, 1 health facility lacked stethoscope. Twelve $(57.2 \%)$ of the facilities had functional thermometer and 4 and 5 had nonfunctional and lacked thermometer respectively. Of the total health facilities surveyed, $5(23.8 \%)$ nonfunctional Blood Pressure (BP) apparatus. Nineteen $(90.5 \%)$ of facilities had no oxygen concentrator and only 3 of the surveyed facilities had functional oxygen cylinder.

\section{Infection Prevention/precautions supplies:} Among 21 surveyed facilities $6(28.6 \%)$ of the facilities had nonfunctional electric autoclave and non-electric autoclave was only found in $2(9.5 \%)$ of facilities. Moreover, 6 health facilities lacked
September 2016 electric dry heat sterilizer. Ten $(47.6 \%)$ of the facilities had clean running water. During the survey, soup for hand washing and alcohol based hand rub was not available in $12(57.1 \%)$ and $5(23.8 \%)$ of the health facilities.

Disposable gloves, waste receptacle and safety box were available in all the health facilities surveyed. From the total health facilities, eye goggle and medical mask were not available in $14(66.7 \%)$ and $9(42.9 \%)$ of the health facilities respectively. Moreover, only $4(19.0 \%)$ of the health facilities were using incineration system of waste disposal.

Laboratory services: All health facilities surveyed conduct malaria diagnosis and urinalysis both dip-stick and microscopic. However, hemoglobin test was not available in $12(57.1 \%$ ) of the health facilities. Syphilis rabid test was available in $19(90.4 \%)$ of the health facilities, but tuberculosis microscopic test and HIV test were not available in $8(38.1 \%)$ and $3(14.3 \%)$ of the health facilities respectively (Table 1 ).

Table 1: Availability of laboratory services among public health facilities of Jimma zone, March, 2015

\begin{tabular}{llll}
\hline Availability of laboratory services/tests & & Frequency & Percent \\
\hline Hemoglobin/ Hematocrit & No & 9 & 42.9 \\
Blood glucose/ Random or Fasting & Yes & 12 & 57.1 \\
Malaria diagnosis /Blood Film & No & 13 & 61.9 \\
& Yes & 8 & 38.1 \\
Urinalysis/ microscopic & No & 0 & 0.0 \\
& Yes & 21 & 100.0 \\
HIV test & No & 0 & 0 \\
& Yes & 21 & 100.0 \\
TB microscopy, & No & 3 & 14.3 \\
& Yes & 18 & 85.7 \\
VDRL for syphilis & No & 8 & 38.1 \\
\multirow{2}{*}{ General microscopy } & Yes & 13 & 61.9 \\
& No & 2 & 9.5 \\
Urine pregnancy test/HCG & Yes & 19 & 90.5 \\
& No & 10 & 47.6 \\
Alanine Aminotransferase (ALT) & Yes & 11 & 52.4 \\
& No & 10 & 47.6 \\
Creatinine & Yes & 11 & 52.4 \\
& No & 19 & 90.5 \\
& Yes & 2 & 9.5 \\
& No & 19 & 90.5 \\
\hline
\end{tabular}

amithriptiline, atenolol and captopril were not

Essential medicines:Among the 14 essential medicines, at the time of the survey, available in $15(71.4 \%)$ of the health facilities. Moreover, hydralazine $25 \mathrm{mg}$ tablet/capsule in 
21(100\%), Ceftriaxone in 3 (14.3\%), Glebinclamide in $4(19.0 \%)$ sulbutamol inhaler in $6(28.6 \%)$ and Diazepam capsule in 7 (33.3\%) of

the health facilities were not available. However, Amoxacilline capsule, cotrimoxazole, ciprofloxacilline, diclofenec, omeprazole, and paracetamol were available in all surveyed health facilities.

Availability of facility governing and health insurance schemes coordinating structures: All of the surveyed facilities had governing board out of which $18(85.7 \%)$ had regular meeting.

Twenty $(95.2 \%)$ of the facilities had management committee which have regular meeting on two weeks base and report activities regularly. Fifteen
(71.4\%) of the facilities were informed about health insurances implementation. Despite the fact, only 1 (4.8\%) of facilities had unit/departments to coordinate Health Insurance schemes.

Overall facility readiness: Among 21 facilities, 8 fulfilled the standard for staffing criteria, 8 for basic infrastructural amenities indicators, 18 for basic equipment, 10 for Infection Prevention/precautions, 2 for laboratory services indicators and 13 for essential medicines indicators. Accordingly, only 2 of the facilities fulfilled the criteria and were judged to be ready to provide general services according to the standard (Table 2).

Table 2: level of availability of variables studied by percentages and counts, Jimma, March, 2015 ( $\mathrm{n}=21$ )

\begin{tabular}{llr}
\hline \multicolumn{1}{c}{ Variables } & Categories & No (\%) of health facility \\
\hline Availability of basic amenities & $<75 \%$ & $13(61.9)$ \\
& $>=75 \%$ & $8(38.1)$ \\
Availability of basic equipment & $<75 \%$ & $3(14.8)$ \\
& $>=75 \%$ & $18(85.2)$ \\
Availability of IP precautions and supplies & $<75 \%$ & $11(52.3)$ \\
& $>=75 \%$ & $10(47.7)$ \\
Availability of laboratory services & $<75 \%$ & $19(90.4)$ \\
& $>=75 \%$ & $2(9.6)$ \\
Availability of essential medicines & $<75 \%$ & $8(38.1)$ \\
& $>=75 \%$ & $13(61.9)$ \\
Availability of facility governing board & Yes & $21(100)$ \\
& No & 0 \\
Availability of board meeting & Yes & $18(85.7)$ \\
& No & $3(14.3)$ \\
Availability of management committee & Yes & $20(95.2)$ \\
& No & $1(4.8)$ \\
Regular meeting of mgt committee & Yes & $20(95.2)$ \\
Informed about implementation of health insurance. & Yes & $1(4.8)$ \\
& No & $15(71.4)$ \\
Unit to coordinate Health Insurance schemes & Yes & $6(28.6)$ \\
& No & $1(4.8)$ \\
& & $20(95.2)$ \\
\hline
\end{tabular}

\section{DISCUSSION}

This study found that the readiness of public health facilities to implement the newly proposed health insurance schemes in Jimma Zone was low by using multiple variables that comprised of seven indicators. Accordingly, the availability of health professionals who can provide general out- patient services lacked appropriate health professionals mix; nearly 4 in 10 health facilities compared to the national standard. The current finding was not in line with the national standard which depicts that there should be optimum number and professional mix of human resource for the effective coverage and quality of the 
454 Ethiop J Health Sci.

intended services (5). The possible explanation

for the observed discrepancy might be that the current study was based on both urban and rural health facilities where the latter had high staff turnover. Furthermore, in this study, the majority of the health facilities which were below standard with health professionals mix were located in rural communities. This finding resembles a report of the national statistics office of the Philippines and the health sector development program I report of Ethiopia which showed that healthcare providers were heavily concentrated in urban and wealthy communities (7).

The quality of the health services available to the insured population is critical to the success of health insurance systems. Meanwhile, the availability of basic infrastructural amenities is crucial for providing quality services that met minimum standards. However, this study showed different phenomena in different facilities. Accordingly, only 4 in 10 health facilities fulfilled the criteria of basic infrastructural amenities. This finding is lower than the finding of a study done on assessment of health facility readiness to provide family planning services in Ghana that showed that $90 \%$ of the facilities met the criteria for infrastructure. The difference might be due to increased infrastructural amenity standard used for analysis and weak health system of the setting of this study (12).

In this study, all the facilities surveyed had power supply from main electric sources. However, only one-third of the facilities had secondary source of electricity, generator, of which only one-half of the facilities had fuel for its operation. These imply that since main power supply line had interruption, there should be secondary sources which are ready for 24 hours a day. When this finding is compared with the findings of SSA which showed that $26 \%$ of the facilities had electricity (13), it was high. This might be due to the fact that the current study used primary data from small sample size.

Communication equipment such as telephone/mobile, computer and internet are important for every health facility. In contrast to this, the current study showed that only 6 in 10 health facilities had fixed line telephone and all had computers, but only 1 in 10 facilities had internet access. Moreover, water supply should be available for facilities to provide quality services. All of the facilities surveyed had water sources at
September 2016

a distance of less than 500 meters even-though it was from different sources. This finding is higher than the findings about water supply of other African regions health facilities, which was only $58 \%$, had an improved water source(11). The possible explination for the observed discrepancy was due to the current study focused on all types of water sources.

Emergency transportation is also important to save life in cases of emergency by faciliatint travel to health facilities. In contrast, less than one half of the facilities surveyed had emergency transport/ambulances stationed at the facilities. One-fourth of the facilities had ambulances stationed at district levels. Patient privacy and enough sanitation/latrine facilities are needed for patient safety and satisfaction. Similarly, in this survey, over three-fourth of the facilities surveyed had consultation rooms with visual and auditory privacy.

Even though all the basic equipment should be available in all facilities to provide minimum standard quality services. In this study, 1 out of 7 health facilities had lower standards. When we compare this finding with the national report of Uganda (14), the current finding was much higher. This difference might be due to the decreased number of basic equipment used as standards in the current study.

Infection prevention supplies and standard precautions should be available in all facilities to protect staffs, patients and other visitors from unwanted infection, while providing services. Thus, at least, sterilization equipment, safe disposal of sharps and infectious wastes, sharps box, waste receptacle, disposable syringes, disinfectant, hand-washing soap and water or alcohol based hand rub, latex gloves, masks, gowns, eye protection, and infection prevention guidelines should be available in every facility. However, this study showed different findings. For example, more than half of the surveyed facilities had below standard required for healthcare facilties.

Even though equipment processing and sterilization should be undertaken before reuse, only three-fourth of the facilities had functional electric autoclave. Moreover, non-electric autoclave was only present in one-fourth of facilities. On the other hand, washing hands with soup before and after any procedure is crucial for infection prevention. However, only one-half of 
the facilities had clean running water with soup for hand-washing. This is lower than the WHO report of African regions health facilities which was $65 \%$ (11).

Effective waste disposal/management system also had important effect on infection prevention. To do so, all the facilities surveyed had safety box/sharp container and medical waste container. However, only $4(19 \%)$ of these facilities incinerate sharp wastes, while the rest use open field burning. These finding was far lower than the finding of a study done in Ethiopia which showed that $40 \%$ of the health centers used incinerators, while others used open burning (17).

The availability of adequate laboratory services is important to diagnose different communicable and non-communicable diseases. However, this study showed discrepancy concerning with this fact. For example, the availability of 13 selected laboratory services was found to be below $75 \%$ in more than $90 \%$ of the facilities surveyed. Furthermore, this study showed laboratory services which are used for diagnosis of non-communicable diseases were less available than laboratory services for diagnosis of communicable diseases. These conditions imply that communicable diseases received more focus than non-communicable diseases although the prevalence of non-communicable diseases is rising.

This study showed that more than one-third of the facilities surveyed had below $75 \%$ of the 14 selected essential medicines, and less than twothird of the surveyed facilities had above $75 \%$ of selected essential medicines. This finding differs from the WHO recommendation which found that all health facilities should have at least those 14 selected essential medicines. Furthermore, the findings of this study was higher than the national report of Uganda that showed only $26 \%$ of availability (15). This difference might be due to the small sample size used in the current study. On the other hand, the current finding is lower than the finding of a study done in Sudan by using WHO operational packages and 30 types of essential medicines and found to be $80.6 \%$ (16).

In this study, the availability of the facility governing board and the management committee that have regular meeting, reporting system and health insurance schemes coordination were assessed. According to the Ethiopian healthcare financing reform, every public health facility should be governed by higher officials called health facility governing board. Moreover, this reform stated that there should be management committee that has regular meeting for discussing and solving facility issues. Accordingly, the majority $(85 \%)$ and more than $95 \%$ of the facilities participated in this survey had governing board and management committee that that have regular meeting respectively. Concerning health insurance schemes, coordination and information disseminations, this study showed that more than 7 in 10 of the facilities surveyed had been informed about the implementation of health insurances. On the contrary, only one facility had unit/department to coordinate the schemes at facility level.

This study had limitations due to the absence of international standards to assess facility readiness. Lowering or increasing the national standard through time can change the interpretation of this data. However, it is concluded that 9 out of 10 facilities did not fulfill the criteria and were not ready to provide general services according to the standard. Hence, policy makers and implementers should devise strategies to fill the identified gaps for successful and sustainable implementation.

\section{ACKNOWLEDGEMENTS}

The authors would like to thank all of the participants of the survey for taking part in this study and Jimma University for funding the research.

\section{REFERENCES}

1. World Health Organization.Universal Coverage and Social Health Insurance. Fifty seventh World Health Assembly: WHO; 2005.

2. Cutler D ZR. The anatomy of health insurance.2000: 563-73

3. Joseph P. Free for all lessons from the Rand health insurance experiment. Harvard University Press; 1993.

4. Chapel H N. Measure Evaluation Health Facility Assessment Technical Working Group. 2007. Available from: http://www.cpc.unc.edu/measure/publications/ pdf/wp-07-97.pdf. .

5. WHO. Working together for health. 2006. 
$456 \quad$ Ethiop J Health Sci.

6. CNN health. Losing Ground. Physician Income. Economic Intelligence Unit Database: world bank 30 global issue; 2013.

7. Office NS. National Demographic andoo Health Survey 2008. Calverton, Maryland: National Statistics Office and ICF Macro; 2009: 176-86.

8. Matsushima M. Public Health Insurance in Vietnam towards Universal Coverage. OSIPP Veitnum; 2013: 15-8.

9. WHO. World Health Statistics. 2012. Available from: http://apps.who.int/iris/bitstream/10665/44844 /1/9789241564441_eng.pdf

10. Uganda Ministry of health. Annual Health Sector Performance Report. Kampala: Ministry of health; 2012.

11. WHO. UN-Water global analysis and assessment of sanitation and drinking-water. Geneva: World Health Organization; 2014.

12. Stephanie H MH, Rafael F. Assessing Facility Readiness of Family Planning Services in Ghana. In press; 2007.

13. Adair RH. Limited electricity access in health facilities of sub-Saharan Africa. Glob Health Sci Pract; 2013:1(2):249-61.
Vol. 26, No. $5 \quad$ September 2016

14. Health Mo. Heath Sector Strategic Plan, 2010/11-2014/15. Kampala, Uganda: MOH; 2010.

15. Uganda. Annual Health Sector Performance Report. Kampala: MoH; 2009.

16. Elfatih I. Availability of Essential Medicines in Sudan. Sudanese Journal of public Health; 2010; 5(1): 34-7.

17. Muluken A AK. Healthcare waste generation and its management system: the case of health centers in West Gojjam Zone, Amhara Region,Ethiopia. Ethiop J Health Dev; 2007.

18. Food, Medcine and Healthcare Adminstration and Control Authority. Ethiopian standards. Addis Ababa: FMHACA; 2012.

19. World Health Organization. Measuring services availablity and readiness. Geneva: WHO 2012.

20. Jayanna K. Assessment of facility readiness and provider preparedness for dealing with postpartum haemorrhage and pre-eclampsia. BMC pregnancy and childbirth. 2014;14:304. 